\title{
Simulating Disk Galaxies: First Results of a Systematical Study
}

\author{
Franziska Köckert and Matthias Steinmetz \\ Astrophysikalisches Institut Potsdam, An der Sternwarte 16, 14482 Potsdam, Germany \\ email: fkoeckert@aip.de
}

Simulating disk galaxies within the current paradigm of galaxy formation has been a long standing problem. In comparison with observations, the simulated disks were too small and too centrally concentrated, due to a large loss of angular momentum during formation. This is known as the angular momentum catastrophe (Navarro \& Benz (1991)). Recently, some progress has been made in reducing this effect by changing the cosmology, including various feedback mechanisms, improving numerical resolution and carefully selecting initial conditions with a quiet merging history after $\mathrm{z} \approx 2$. Unfortunately, it remains unclear which of these effects, or which combination, has resulted in more realistic disk formation. In order to address this problem, we conduct a systematical study using the N-body code GADGET2 (Springel (2005)). We adopt a flat $\Lambda$ CDM cosmology with $\Omega_{m}=0.3, \Omega_{\Lambda}=0.7, \Omega_{b a r}=0.04$ and $\mathrm{h}=0.65$. Using a softening of $0.5 \mathrm{kpc}$ we find disks with a very compact unresolved gas clump in the center and a thin, extended disk $(\mathrm{R} \approx 10 \mathrm{kpc})$ of very low mass around it.

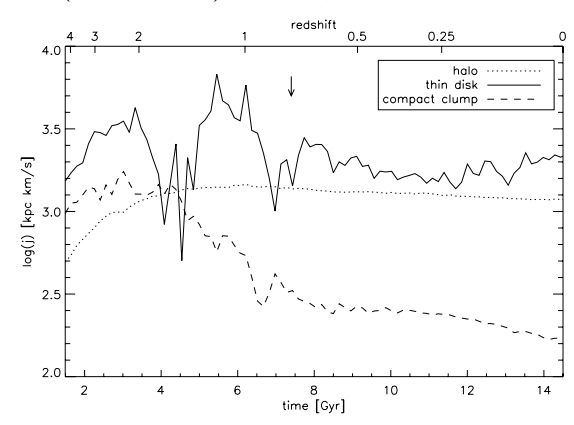

Figure 1. The time evolution of the specific angular momentum of the dark matter halo (dotted), the thin disk (solid) and the compact clump (dashed). The arrow indicates the time of the last bigger merger.

The reason for this is the loss of angular momentum, which according to our findings happens during the collapse of the disk and is not simply due to numerical effects. The compact component experiences a great loss of angular momentum in the last bigger merger at around redshift 0.7. After that, the angular momentum of both the compact and the thin component stays fairly constant (see figure). Contradicting previous findings (Navarro \& Steinmetz (1997)), we find that including the UV background increases the angular momentum the particles manage to retain until $\mathrm{z}=0$.

Including star formation according to a recipe from Mihos \& Hernquist (1996) results in a very small gas disk which is traced by a disk of young stars $(<4 \mathrm{Gyr})$. However, compared to observations too many stars are formed overall.

We plan to extend our study to include more sets of initial conditions, also at different resolutions. As a next step we will look at different star formation recipes and also include feedback mechanisms.

\section{References}

Mihos, J.C., \& Hernquist, L. 1996, ApJ 464, 641.

Navarro, J.F., \& Benz, W. 1991, ApJ 380, 320.

Navarro, J.F., \& Steinmetz, M. 1997, ApJ 478, 13.

Springel, V. 2005, MNRAS 364, 1105S. 